\title{
Depressão entre acadêmicos de enfermagem e os fatores sociodemográficos associados
}

\author{
Depression among nursing students and associated sociodemographic factors
}

Depresión en estudiantes de enfermería y factores sociodemográficos associados

Liliane Santos da Silva ${ }^{1 *}$, Maria Aparecida Sousa Oliveira Almeida ${ }^{1,2}$, Elias Marcelino da Rocha ${ }^{1}$, Rosa Jacinto Volpato ${ }^{1}$, Pâmela Roberta de Oliveira ${ }^{1}$, Vagner Ferreira do Nascimento ${ }^{3}$, Alisséia Guimarães Lemes ${ }^{1,2}$.

\section{RESUMO}

Objetivo: O presente estudo buscou identificar a presença de depressão e os fatores sociodemográficos associados a esse transtorno entre acadêmicos de enfermagem. Métodos: estudo transversal, descritivo quantitativo, realizado como 60 acadêmicos do curso de enfermagem de uma universidade pública no interior de Mato Grosso no ano de 2016 que foram avaliados por meio de um questionário semiestruturado e o Inventário de Depressão de Beck (BDI). Resultados: a análise dos resultados demostrou que a tendência a depressão esteve presente em $46,66 \%$ da amostra, com intensidade leve, moderada e grave, havendo uma forte associação dos sintomas depressivos com a faixa etária e uma associação de média intensidade com o estado civil, renda mensal individual, religião e arranjo familiar. Conclusão: os resultados demonstram a necessidade de um rastreamento contínuo dos sintomas depressivos e apoio aos acadêmicos em risco, minimizando assim o sofrimento mental nesse grupo de pessoas.

Palavras-chave: Depressão, Estudantes de enfermagem, Saúde Mental.

\begin{abstract}
Objective: The present study aimed to identify the presence of depression and the sociodemographic factors associated with this disorder among nursing students. Methods: cross-sectional, quantitative descriptive study conducted with 60 undergraduate nursing students from a public university in the interior of Mato Grosso in 2016 who were assessed using a semi-structured questionnaire and the Beck Depression Inventory (BDI). Results: The analysis of the results showed that the tendency to depression was present in $46.66 \%$ of the sample, with mild, moderate and severe intensity, with a strong association of depressive symptoms with age and an association of medium intensity with marital status. individual monthly income, religion and family arrangement. Conclusion: The results demonstrate the need for continuous tracking of depressive symptoms and support for at-risk academics, thus minimizing mental distress in this group of people.
\end{abstract}

Key words: Depression, Nursing students, Mental health.

\footnotetext{
1 Universidade Federal de Mato Grosso (UFMT), Campus Universitário do Araguaia (CUA). Barra do Garças, MT, Brasil. *E-mail: liliane rodrigues23@hotmail.com

${ }^{2}$ Escola de Enfermagem de Ribeirão Preto da Universidade de São Paulo (EERP-USP), Ribeirão Preto, SP, Brasil.

${ }^{3}$ Universidade do Estado de Mato Grosso (UNEMAT). Tangará da Serra, MT, Brasil.
} 


\section{RESUMEN}

Objetivo: El presente estudio tuvo como objetivo identificar la presencia de depresión y los factores sociodemográficos asociados con este trastorno entre los estudiantes de enfermería. Métodos: estudio descriptivo, cuantitativo y transversal realizado con 60 estudiantes universitarios de enfermería de una universidad pública del interior de Mato Grosso en 2016 que fueron evaluados mediante un cuestionario semiestructurado y el Inventario de depresión de Beck (BDI). Resultados: El análisis de los resultados mostró que la tendencia a la depresión estaba presente en el $46,66 \%$ de la muestra, con intensidad leve, moderada y severa, con una fuerte asociación de síntomas depresivos con la edad y una asociación de intensidad media con el estado civil. ingreso mensual individual, religión y arreglo familiar. Conclusión: Los resultados demuestran la necesidad de un seguimiento continuo de los síntomas depresivos y el apoyo a los académicos en riesgo, minimizando así la angustia mental en este grupo de personas.

Palabras clave: Depresión, Estudiantes de enfermería, Salud mental.

\section{INTRODUÇÃO}

A depressão é considerada um transtorno mental de causa multifatorial, que atinge mais de 300 milhões de pessoas de todas as idades, causando incapacidades, sofrimento, alterações no convívio social, e na pior das hipóteses o suicídio. Embora já seja conhecido vários tratamentos eficazes para a depressão, devido à falta de recursos, de profissionais qualificados, diagnósticos incorretos, estigma social, entre outros obstáculos, poucas pessoas têm acesso a esses tratamentos (WHO, 2018).

A doença não se restringe a ciclos de vida nem a espaços geográficos, mas nos últimos anos, com o acesso à tecnologia e comprometimento das relações interpessoais, parece que os sinais e sintomas estão surgindo precocemente. No ambiente universitário, essas demandas psicossociais tendem a ser ampliadas e comprometer tanto o desempenho do estudantes como sua qualidade de vida (BAUER TX, et al., 2018).

A saúde mental em equilíbrio na graduação favorece o desenvolvimento acadêmico e o aprendizado. Nesse momento se inicia uma etapa fundamental para o crescimento pessoal e profissional do indivíduo, a qual será percebida e vivenciada por eles de diferentes maneiras (VIZZOTO MM, et al., 2017). Esse período pode ser marcado por mudanças, as quais irão exigir maior responsabilidades e maturidade, pois além dos desafios em lidar na maioria das vezes com mudança de cidade e distanciamento dos pais, familiares e amigos, existe as pressões vivenciadas no ambiente acadêmico, que exigirá do universitário habilidades interpessoais e acadêmicas (VADEBONCOEUR C, et al., 2015; BOLSONI-SILVA AT e GERRA BT, 2014; MESQUITA AM, et al., 2016).

Aproximadamente $15 \%$ a $25 \%$ dos acadêmicos desenvolvem transtornos mentais na graduação, destacando-se a depressão (SANTOS KFR, et al., 2018). O desenvolvimento de depressão em estudante de enfermagem pode estar associado a sentimentos desenvolvidos no processo de ensino-aprendizagem, por ser um curso que lida com vidas (CAMARGO RM, et al., 2014).

Esse cenário estressor pode contribuir para o agravamento de doenças mentais já existentes ou colaborar para que a mesma se desenvolva (BOLSONIR-SILVA AT e GERRA BT, 2014). Sendo um assunto que desde os anos 60 já era motivo de preocupações e debates nos Estados Unidos, onde criou-se a primeira assistência estudantil em saúde mental, ao compreender a gravidade do problema e a importância das instituições de ensino como suporte (CERCHIARE EAN, et al., 2005).

É importante destacar que existe estratégias de prevenção a depressão que proporciona resultados satisfatórios, uma vez que a doença seja em níveis leve, moderado ou grave de acordo com a intensidade dos sintomas, causam prejuízos a vida do indivíduo (ROCHA-ALMEIDA LG e FARO A, 2016, WHO, 2018).

Diante o exposto, identificar os fatores de risco para o adoecimento mental em universitários é uma estratégia importante para o desenvolvimento de ações de promoção, proteção e prevenção em saúde dentro das universidades. Neste sentido, o objetivo desse estudo foi identificar a presença de depressão e os fatores sociodemográficos associados a esse transtorno entre acadêmicos de enfermagem. 


\section{MÉTODOS}

Trata-se de um estudo transversal, descritivo quantitativo, realizado com acadêmicos de enfermagem de uma universidade pública no interior de Mato Grosso, que atenderam os critérios estabelecidos para este estudo.

Os critérios de inclusão foram: ter idade $\geq 18$ anos e estar regularmente matriculado no curso de enfermagem.

Os critérios de exclusão foram: acadêmicos que não estiveram presentes na sala de aula no momento da coleta de dados ou que se encontraram afastados por atestado médico e/ou licença maternidade.

Para a definição da amostra, utilizou uma amostragem por conveniência, dos 133 acadêmicos de enfermagem matriculados nesta Instituição de Ensino Superior (IES).

O curso de enfermagem nesta universidade ocorre de forma integral (matutino e vespertino) e possui uma grade curricular em $8^{\circ}$ semestres. Deste total, aceitaram participar do estudo 64 estudantes.

Houve perdas de quatro questionários, por preenchimento incompleto, totalizando assim, uma amostra de 60 estudantes.

Os dados foram coletados no período de julho a outubro de 2016, por meio da aplicação de um questionário semiestruturado, contendo questões objetivas, sobre os dados sociodemográficos (faixa etária, sexo, estado civil, renda individual mensal, religião e arranjo familiar) e o Inventário de Depressão de Beck (BDI) que trata-se de um questionário validado, desenvolvido para investigar a presença e a intensidade da depressão, contendo 21 itens, com escala tipo likert $(0,1,2,3)$, podendo pontuar de 0 a 63 pontos, sendo considerado como ponto de corte de 0-11 pontos sinalizando ausência de depressão, 12-19 pontos depressão leve, 20-35 pontos depressão moderada e 36-63 pontos depressão grave (CUNHA JA, 2016).

Os participantes responderam os instrumentos da pesquisa nas salas de aula, após permissão do docente e preenchimento do Termo de Consentimento Livre e Esclarecido (TCLE). A aplicação dos instrumentos teve a duração média de 30 minutos.

Os dados foram lançados e organizados no programa Microsoft Office Excel 2013, posteriormente, foram processados e analisados no programa Epiinfo versão 3.5.2 e Bioestat versão 5.0. A estatística descritiva foi aplicada nas variáveis sociodemográficas, semestres do curso, presença de depressão.

Foi realizado um cruzamento descritivo entre as variáveis sociodemográficas e a presença de depressão e para avaliar as possíveis associações da ideação suicida entre essas variáveis foi utilizado o programa BioEstat versão 5.0, por meio da análise de correlação "Coeficiente de Contingência C" (resultado de C=0, determina que não há associação entre as variáveis, quando $C \neq 0$, há correlação entre duas variáveis $-<0.1$ fraca, 0.1 a 0.3 moderada e >0.3 forte; sendo adotado o nível de significância de $5 \%$ - $p<0,05$ ).

Todas as providências em relação à dimensão ética do estudo foram tomadas de acordo com a Resolução de ética 466/2012.

O estudo foi aprovado pelo Comitê de Ética em Pesquisa da UFMT/CUA, sob o protocolo no 515/705.

\section{RESULTADOS}

Participaram do estudo 60 acadêmicos de enfermagem, com idade entre 18 e 37 anos e média de 22 anos, predominando acadêmicos com idade entre 18 a 22 anos (72\%), do sexo feminino (80\%), solteiros (82\%), com renda mensal de até 1 salário mínimo $(60 \%)$, sem ocupação remunerada $(80 \%)$, com algum tipo de religião (83\%), residindo com o pai e/ou mãe (35\%) e sozinho (30\%).

O uso do BDI revelou que $46,66 \%$ dos sujeitos apresentaram algum nível de depressão, com destaque para o tipo leve, presente entre $35 \%$ dos acadêmicos (Gráfico 1). 
Gráfico 1 - Distribuição dos níveis de Depressão entre os acadêmicos de enfermagem. Barra do Garças, MT, Brasil, 2016. $(n=60)$

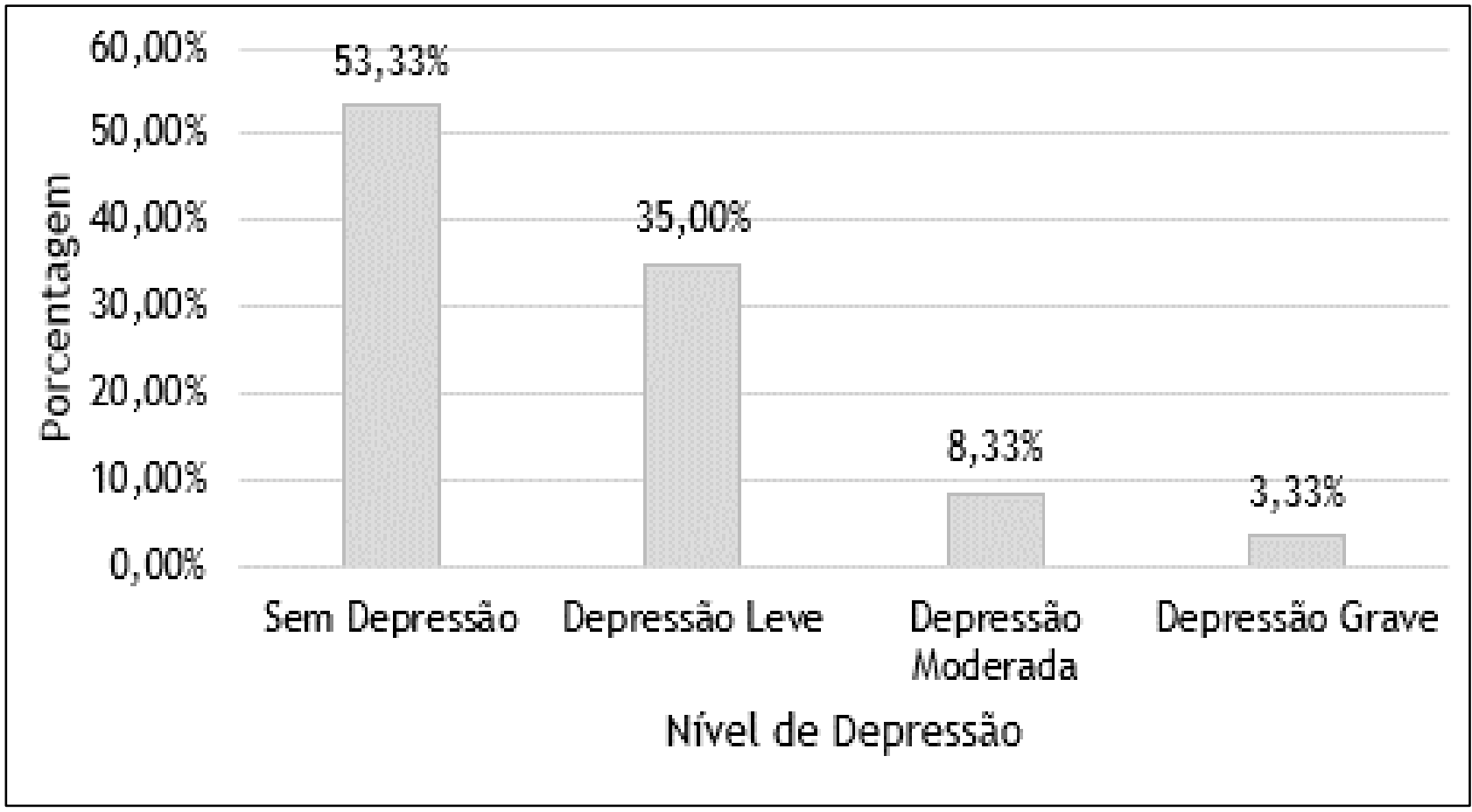

Fonte: Silva LS, et al., 2016.

A depressão esteve presente entre os alunos de todos os semestres do curso de enfermagem, com destaque para os casos leve e moderado apresentados no $5^{\circ}$ e $7^{\circ}$ semestre e de casos graves apresentado no 3 o semestre (Tabela 1).

Tabela 1 - Distribuição dos semestres cursado com a presença de depressão entre os acadêmicos de enfermagem. Barra do Garças, MT, Brasil, 2016. ( $n=60)$

\begin{tabular}{|c|c|c|c|c|c|}
\hline \multirow[b]{2}{*}{ Semestre } & \multicolumn{5}{|c|}{ Nível de Depressão } \\
\hline & $\begin{array}{c}\text { Sem } \\
\text { Depressão }\end{array}$ & $\begin{array}{c}\text { Depressão } \\
\text { Leve }\end{array}$ & $\begin{array}{l}\text { Depressão } \\
\text { Moderada }\end{array}$ & $\begin{array}{c}\text { Depressão } \\
\text { Grave }\end{array}$ & Total \\
\hline & $\mathrm{N}(\%)$ & $\mathrm{N}(\%)$ & $\mathrm{N}(\%)$ & $\mathrm{N}(\%)$ & $\mathbf{N}(\%)$ \\
\hline 1ㅇ Semestre & $09(15 \%)$ & $03(5 \%)$ & $01(2 \%)$ & $00(00)$ & $13(22 \%)$ \\
\hline $3^{\circ}$ Semestre & $13(22 \%)$ & $04(7 \%)$ & $00(00)$ & $02(3 \%)$ & $19(31 \%)$ \\
\hline $5^{\circ}$ Semestre & $03(5 \%)$ & $06(10 \%)$ & $03(5 \%)$ & $00(00)$ & $12(20 \%)$ \\
\hline $7^{\circ}$ Semestre & 05 (9\%) & $08(13 \%)$ & $00(00)$ & $00(00)$ & $13(22 \%)$ \\
\hline $8^{\circ}$ Semestre & $02(3 \%)$ & $00(00)$ & $01(2 \%)$ & $00(00)$ & $03(5 \%)$ \\
\hline Total & $32(54 \%)$ & $21(35 \%)$ & $05(8 \%)$ & $02(3 \%)$ & $60(100 \%)$ \\
\hline
\end{tabular}

Fonte: Silva LS, et al., 2016.

Ao associar as variáveis do sociodemográficas com a depressão, verificou-se que houve uma forte associação entre faixa etária $(C=0,347 ; p=0,041)$ com esse transtorno mental. Destacando ainda, uma associação de média intensidade entre a depressão com o estado civil $(C=0,163)$, com a renda mensal individual $(C=0,142)$, com a religião $(C=0,204)$ e com o arranjo familiar $(C=0,170)$ (Tabela 2). 
Tabela 2 - Distribuição da associação da depressão com as variáveis sociodemográficas entre os acadêmicos de enfermagem. Barra do Garças, MT, Brasil, 2016. $(n=60)$

\begin{tabular}{|c|c|c|c|c|}
\hline \multirow[t]{2}{*}{ Variáveis } & \multicolumn{2}{|c|}{ Presença de Depressão } & \multicolumn{2}{|l|}{ Estatística } \\
\hline & Sim & Não & ${ }^{*}$ Teste de Contingência & $p$-valor \\
\hline \multicolumn{5}{|l|}{ Faixa etária } \\
\hline 18 a 22 anos & $20(71 \%)$ & $23(72 \%)$ & & \\
\hline 23 a 27 anos & $8(29 \%)$ & $3(10 \%)$ & $0,347^{* * *}$ & $0,041^{* * * *}$ \\
\hline 28 a 32 anos & $0(0 \%)$ & $4(12 \%)$ & & \\
\hline 33 a 37 anos & $0(0 \%)$ & $2(6 \%)$ & & \\
\hline \multicolumn{5}{|l|}{ Sexo } \\
\hline Feminino & $22(79 \%)$ & $26(81 \%)$ & 0,033 & 0,948 \\
\hline Masculino & $6(21 \%)$ & $6(19 \%)$ & & \\
\hline \multicolumn{5}{|l|}{ Estado Civil } \\
\hline Solteiro & $22(79 \%)$ & $27(84 \%)$ & & \\
\hline Casado/União estável & $6(21 \%)$ & $4(13 \%)$ & $0.163^{* *}$ & 0,438 \\
\hline Separado & $0(0 \%)$ & $1(3 \%)$ & & \\
\hline \multicolumn{5}{|l|}{ Renda mensal individual } \\
\hline <1 salário Mínimo & $15(54 \%)$ & $21(66 \%)$ & & \\
\hline 1 a 2 salário Mínimo & $5(18 \%)$ & $3(9 \%)$ & $0.142^{* *}$ & 0,743 \\
\hline >3 salário Mínimo & $1(3 \%)$ & $1(3 \%)$ & & \\
\hline Não Respondeu & $7(25 \%)$ & 7 (22\%) & & \\
\hline \multicolumn{5}{|l|}{ Ocupação remunerada } \\
\hline Sim & $6(21 \%)$ & $6(19 \%)$ & 0,033 & 0,948 \\
\hline Não & $22(79 \%)$ & $26(81 \%)$ & & \\
\hline \multicolumn{5}{|l|}{ Possui religião } \\
\hline Sim & $21(75 \%)$ & $29(91 \%)$ & $0,204^{* *}$ & 0,203 \\
\hline Não & $7(25 \%)$ & $3(9 \%)$ & & \\
\hline \multicolumn{5}{|l|}{ Arranjo familiar } \\
\hline Pai e/ou mãe & $8(29 \%)$ & $13(41 \%)$ & & \\
\hline Sozinho & $9(32 \%)$ & $9(28 \%)$ & & \\
\hline Outros familiares & $5(18 \%)$ & $5(16 \%)$ & $0,170^{* *}$ & 0,876 \\
\hline Amigos & $3(11 \%)$ & $2(6 \%)$ & & \\
\hline Conjugue & $2(7 \%)$ & $1(3 \%)$ & & \\
\hline Em republica & $1(3 \%)$ & $2(6 \%)$ & & \\
\hline
\end{tabular}

${ }^{{ }^{C} \mathrm{C}=0}$ - Não há associação entre as variáveis; $\mathrm{C} \neq 0$ - há correlação entre duas variáveis $\left(<0.1\right.$ fraca, ${ }^{\star \star} 0.1$ a 0.3 moderada $e^{* * *}>0.3$ forte) ${ }^{* * * *} p<0,05$.

Fonte: Silva LS, et al., 2016. 


\section{DISCUSSÃO}

Neste estudo a depressão esteve presente entre os acadêmicos de enfermagem, com destaque para o tipo leve da doença. Corroborando com os achados de presença de depressão leve entre os universitários, encontrados em pesquisas nacionais realizada em Mato Grosso, São Paulo, Brasília, Piauí, Ceara, assim como identificado em pesquisas internacionais, realizada na Índia, Arábia Saudita e na Turquia (CAMARGO RM, et al, 2014; CHATTERJEER S, et al., 2014; MESQUITA AM, et al., 2016; POLONIO IB, et al., 2017; YOUSEF S, et al., 2017; FERNANDES MA, et al., 2018; LEÃO AM, et al., 2018; YÜKSEL A e BAHADIRYILMAZ E, 2019).

Pode-se observar que a depressão tem sido comumente encontrada entre os acadêmicos, chamando atenção para a necessidade de se promover atividades de rastreio no ambiente universitário com a finalidade de identificar precocemente os casos existentes e ofertar possibilidades de tratamento e o bem-estar psicológico, como o encaminhamento a atividades preventivas e/ou de acolhimento (AZAD N, et al., 2017). Outro tipo de recurso seria direciona-los para participarem de atividades coletivas como a oferta de práticas integrativas e complementares, que tem apresentado bons resultados em diferentes públicos que podem auxiliar no tratamento voltado para depressão, ansiedade e estresse e uso de drogas psicoativas (LEMES AG, et al., 2017; DACAL MDPO e SILVA IS, 2018; BELASCO IC, et al., 2019). Bem como o atendimento psicológico e/ou psiquiátrico nas redes de atenção do município para os casos mais graves, que não seja possível o acolhimento apenas no âmbito da universidade.

Diante da necessidade de se identificar cada vez mais precoce os universitários com depressão, torna-se relevante saber em que fase do curso eles podem ser mais suscetíveis a esse adoecimento. Observou-se um maior percentual de acadêmicos com tendência a depressão entre os dois últimos anos do curso (5ํㅜ e $7^{\circ}$ semestre), assim como também foi verificado entre estudantes dos últimos anos do curso de enfermagem na China (LUO Y, et al., 2019). Isso pode ser devido ao crescente contato nos últimos anos da graduação com pacientes em situações de sofrimento, dor e muitas vezes a morte (MESQUITA AM, et al., 2016).

Porém, um estudo realizado na Índia revelou uma maior prevalência de depressão entre os estudantes do primeiro ano, havendo uma redução gradativa nos anos subsequentes (CHATTERJEE S, et al., 2014). Um estudo reportou que a prevalência de depressão pode variar por semestre de acordo com fatores culturais, sociais e econômicas, assim como a grade curricular e a carga horaria de dedicação aos estudos, que são fatores que mudam conforme a IES e a sociedade em questão (FERNANDES MA, et al., 2018).

Os resultados apontam para uma necessidade urgente da reavaliação dos planos pedagógicos do curso, para o aluno se dedicar ao curso sem adoecimento e assim diminuir a evasão (LIMA VV, et al., 2019). E também propiciar aos universitários ferramentas para o melhorar o enfrentamento das dificuldades encontradas, e dedicar-se a outras atividades voltada ao descanso, lazer, permitindo assim o seu envolvimento em outras atividades intra ou extra muros da universidade (ARIÑO DO e BARDAGI MP, 2018; PARO CA e BITTENCOURT ZZLC, 2013). Desta forma, acredita-se que será possível contribuir com a redução dos sintomas da depressão e de outras doenças mentais entre essa população.

Um outro fator a ser destacado neste estudo é a necessidade de se identificar quais são as características sociodemográficas dos estudantes que apresentam com quadro depressivo. Como pode ser visto, neste estudo houve uma forte associação entre a depressão e a faixa etária, revelando um maior adoecimento entre pessoas jovens.

Pesquisadores referem que essa faixa etária está permeada por um período de maior suscetibilidade, devido as mudanças vivenciadas nesse fase da vida, considerada como um momento de transição para a vida adulta, marcado por escolhas e incertezas que causam instabilidades emocionais, propiciando 0 adoecimento físico e mental (CARDOSO HF, et al., 2018). Por esses motivos, essa faixa etária (pessoas jovens) tem sido apontada como fator de risco de maior relevância para o adoecimento mental, por ser uma fase de desenvolvimento da identidade própria (OSSE CMC e COSTA II, 2011; MATOS NA, 2014). 
Grande parte dos estudantes de cursos integrais fazem parte dessa faixa etária, tendo em vista as peculiaridades que esses cursos exigem, como dedicação integral aos estudos, estágios e outras vivencias, e que por consequência diminui o tempo para atividades de lazer (SILVA JH, et al., 2019). Desta forma, percebe-se a necessidade de compreender melhor esse adoecimento para que seja possível desenvolver atividades que auxiliem na prevenção ou acompanhamento desse grupo de pessoas ao longo da sua permanência na academia. Pois, se não for ofertado cuidado apropriados ocorrerá efeitos indesejados ao longo da vida pessoal e profissional (AZAD N, et al., 2017).

Neste estudo, observou que a depressão esteve mais presente entre estudantes solteiros, assim como também apontado em outros estudos (COSTA CRB, et al., 2018; MESQUITA AM, et al., 2016). Já no estudo realizado na Arábia Saudita o estado civil não foi apontado como fator associado a depressão, estresse ou ansiedade (BASUDAN S, et al., 2017). Sobre isso, é importante destacar que a existência de uma relação afetiva e de suporte social adequado, pode permitir espaço para o diálogo e para o compartilhamento de sentimentos, o que é essencial no processo de superação de eventos estressores, o que pode contribuir para evitar um possível adoecimento mental, como a depressão (COSTA CRB, et al., 2018; GOMES RK e OLIVEIRA VB, 2013).

Verificou-se também a associação da depressão com o baixo nível socioeconômico dos estudantes, permeado pela pouca renda mensal e ausência de um emprego renumerado, condição social também registrada em um estudo realizado na capital mato-grossense (BR) (SANTOS et al., 2018). Um estudo realizado em Caruaru (PE) constatou que a maior parte dos universitários participavam de financiamento e/ou bolsa estudantil (SILVA JH, et al., 2019), subtendendo a necessidade de ajuda financeira para se manterem no estudo.

Autores ressaltam que acadêmicos que apresentem dificuldade financeira tem 2,3 vezes mais propensos a desenvolver depressão (CHEUNG T, et al., 2016). Diante desse contexto é de suma importância que as IES proporcionem suporte financeiro aos universitários, seja pela oferta de bolsas extensão, monitoria, pesquisa ou por meio de auxílios como de moradia e alimentação, podendo assim, contribuir com a permanência desses alunos nos cursos, em especial nos que ocorrem em período integral, por exigirem dedicação exclusiva o que dificulta aos acadêmicos procurarem ou se manterem em emprego renumerado.

Um outro aspecto social identificado neste estudo foi a presença da religião entre os universitários que apresentaram ou não depressão, revelando ainda, uma associação mediana entre essas variáveis (depressão $v s$ religião). A depressão apresentou-se fortemente associada com a religião em um estudo nacional realizado com acadêmicos de medicina em Juiz de Fora, MG (MOUTINHO ILD, et al., 2017), e ausente em um estudo realizado em Uberaba, MG (GONÇALVES JLR, et al., 2018).

Sobre a religião, autores ressaltam essa variável como um fator protetor para a depressão, podendo auxiliar o estudante a superar os problemas enfrentados por eles nesse período (ASANTE KO e ANDOHARTHUR J, 2015; YOUSEF S, et al., 2017; WERNER MEC, et al., 2015).

A OMS destaca que a religião pode ser considerada um fator preventivo ou não. Preventivo, pois a fé, pode encorajar a enfrentar momentos de adversidade ao crer em um ser superior, por proporcionar momentos de interação, compartilhamento de valores, e ao orientar e até mesmo proibir ações ilícitas e o uso de drogas psicoativas. Não preventivo, a partir do momento que algumas religiões tratam o ato suicida como ações demoníacas, desencorajando o fiel a procurar ajuda de um profissional (WHO, 2014).

Neste estudo, houve uma associação mediana entre a depressão e o arranjo familiar, percebeu-se ainda que dos acadêmicos que apresentaram depressão a maioria se autodeclaram morar sozinho. Residir sozinho no período acadêmico também foi reportado em outros dois estudos. O primeiro, uma pesquisa que teve como objetivo averiguar o adoecimento mental entre universitários, revelou que $23 \%$ de seus entrevistados relataram residirem sozinhos (MESQUITA AM, et al., 2016).

O segundo, um levantamento realizado com estudantes de medicina no Amapá, PR, reportou que 17,2\% dos estudantes residiam sozinhos e 17,8\% com outros colegas (OLIVEIRA GS, et al., 2016). 
Sobre isso, destaca-se que morar sozinho e o risco para depressão vai depender de fatores como local e vínculo social que ela vai criar, como exemplo, em residência universitária, o risco de desenvolvimento de adoecimento mental é bem menor (GONÇALVES AM, et al., 2011; PEREIRA AAG e CARDOSO FMS, 2015). Deve-se levar em consideração o fato desses estudantes estarem longe do convívio com a família e longe do seu município, o que pode propiciar a presença de depressão e a ansiedade (VASCONCELOS TC, et al., 2015).

Eisenberg D et al. (2007) refere que residir com o parceiro (casado ou em um relacionamento estável) permite aos estudantes manter maiores níveis de saúde mental e consequentemente reduz o risco para os pensamentos suicidas. Em contrapartida, um estudo reportou sobre o fato de o acadêmico residir sozinho tem sido considerado um fator de risco para transtornos mentais comuns, como a insônia, cefaleia, fadiga, irritabilidade, esquecimento, dificuldade de concentração, tristeza, ansiedade e preocupação somática. Isso justifica-se porque ao morar sozinho, há uma diminuição do apoio emocional do qual estes acadêmicos necessitam (LIMA VV, et al., 2006).

Ao se confirmar que o adoecimento mental esteve mais presente entre pessoas que redizem sozinhos, torna-se necessário a oferta de atividades no meio universitário que inclua esses estudantes em grupo, sendo possível contribuir para ampliar suas relações e vínculos sociais, bem como ofertar um ambiente que possam acolhe-los e oferecer ajuda, de forma que quando for necessário possam retornar em busca de cuidado e apoio. Espera-se que através dos vínculos estabelecidos com colegas, professores e funcionários, possa ser mais fácil identificar precocemente os casos de adoecimento mental, uma vez que os universitários geralmente não conseguem identificar que precisa de ajuda profissional sozinho (SANTOS KFR, et al., 2018).

\section{CONSIDERAÇÕES FINAIS}

O presente estudo permitiu identificar os acadêmicos de enfermagem com depressão e características sociodemográficas que apresentaram associações leve e moderada com esse transtorno mental. Diante os resultados expostos, pode-se observar um percentual significante de depressão leve entre os acadêmicos, principalmente entre os últimos anos da graduação, os mais jovens que residem sozinhos e solteiros. Sendo um alerta de suma importância o despertar da gestão universitária para o desenvolvimento e implementação de programas de intervenções, com estratégias de prevenção, rastreamento e acolhimento da população acadêmica em risco. Incentivando a participação do indivíduo em atividades de interação social no campus e a oferta de oficinas que permita a estes jovens aprender a gerenciar a vida acadêmica. Almejando assim minimizar os danos causados pelo sofrimento mental na graduação, promovendo melhora no desempenho acadêmico e da qualidade de vida dessa população.

\section{REFERÊNCIAS}

1. ARIÑO DO, BARDAGI MP. Relação entre Fatores Acadêmicos e a Saúde Mental de Estudantes Universitários. Revista Psicologia em Pesquisa, 2018; 12(3).

2. ASANTE KO, ANDOH-ARTHUR J. Prevalence and determinants of depressive symptoms among university students in Ghana. Journal of Affective Disorders, 2015; 171: 161-166.

3. AZAD N, et al. Anxiety and depression in medical students of a private medical college. Journal of Ayub Medical College Abbottabad, 2017; 29(1): 123-127.

4. BASUDAN S, et al. Depressão, ansiedade e estresse em estudantes de odontologia. Internacional Journal of Medical Education, 2017; 8: 179-186.

5. BAUER TX, et al. Drogas psicoativas no contexto de trabalho do professor de escola pública. Journal Health NPEPS, 2018; 3(1): 166-184.

6. BELASCO IC, et al. Práticas integrativas e complementares na saúde mental do estudante universitário. Arquivos Brasileiros de Psicologia, 2019; 71(1): 103-111.

7. BOLSONI-SILVA AT, GUERRA BT. O impacto da depressão para as interações sociais de universitários. Estudos e Pesquisas em Psicologia, 2014; 14(2): 429-452.

8. CAMARGO RM, et al. Prevalência de casos de depressão em acadêmicos de enfermagem em uma instituição de ensino de Brasília. Rev Min Enferm., 2014; 18(2): 398-403.

9. CARDOSO HF, et al. Indicadores de saúde mental em jovens: fatores de risco e de proteção. Est. Inter. Psicol. 2018;.9(3): 3-25. 
10. CHATTERJEE S, et al. Depressão entre estudantes de enfermagem em uma faculdade do governo indiano. British Journal of Nursing, 2014; 23(6).

11. CHEUNG T, et al. Depressão, ansiedade e sintomas de estresse entre estudantes de enfermagem de bacharelado em Hong Kong: um estudo transversal. Int J Environ Res Saúde Pública, 2016; 13(8): 779.

12. COSTA CRB, et al. Estresse entre estudantes de graduação em enfermagem: associação de características sociodemográficas e acadêmicas. Saúde e Pesquisa, 2018; 11(3): 475-482.

13. DACAL MDPO, SILVA IS. Impactos das práticas integrativas e complementares na saúde de pacientes crônicos. Saúde em Debate, 2018; 42: 724-735.

14. EISENBERG D, et al. Prevalência e correlatos de depressão, ansiedade e suicídio entre universitários. American Journal of Orthopsychiatry, 2007; 77(4): 534-542.

15. FERNANDES MA, et al. Prevalência de sintomas ansiosos e depressivos em universitários de uma instituição pública. Rev Bras Enferm., 2018; 71(suppl 5): 2298-304.

16. GOMES RK, OLIVEIRA VB. Depressão, ensiedade e suporte social em profissionais de enfermagem. Bol. Psicol., 2013; 63(138).

17. GONÇALVES AM, et al. Comportamentos Suicidários em Estudantes do Ensino Superior: Factores de Risco e de Protecção. Millenium. 2011; (40): 149-159.

18. GONÇALVES JRL, et al. A religiosidade está associada a níveis mais baixos de ansiedade, mas não de depressão, em estudantes de medicina e enfermagem. Rev. Assoc. Med. Bras., 2018; 64(6).

19. LEÃO AM, et al. Prevalência e Fatores Associados à Depressão e Ansiedade entre Estudantes Universitários da Área da Saúde de um Grande Centro Urbano do Nordeste do Brasil. Revista Brasileira de Educação Médica, 2018; 42(4): 55-65.

20. LEMES AG, et al. Integrative Community Therapy as a strategy for coping with drug among inmates in therapeutic communities: documentary research. Rev eletrônica saúde mental alcool drog., 2017; 13(2):101-108.

21. LIMA VV, et al. Repercussão do ambiente universitários em fatores comportamentais, bioquímicos e psicológico no câmpus araguaia. Corixo-Revista de Extensão Universitária, 2019; (7).

22. LIMA MCP, et al. Prevalência e fatores de risco para transtornos mentais comuns entre estudantes de medicina. Revista de Saúde Pública, 2006; 40: 1035-1041.

23. LUO Y, et al. Self-compassion may reduce enxiety end depression in nursing students: a pathway through perceived stress. Public Health, 2019; (174): 1-10.

24. MATOS NA. Conhecendo o sofrimento psíquico dos universitários da faculdade de Ceilândia (Monografia). Brasília: Bacharel em Terapia Ocupacional - Universidade de Brasília, 2014. $69 \mathrm{f}$.

25. MESQUITA AM, et al. Depressão entre estudantes de cursos da área da saúde de uma Universidade de Mato Grosso. Journal Health NPEPS. 2016; 1(2):218-230.

26. MOUTINHO ILD, et al. Depression, stress and anxiety in medical students: A cross--sectional comparison between students from different semesters. Rev Assoc Med Bras. 2017; 63(1): 21-8.

27. OLIVEIRA GS, et al. Prevalência e fatores associados à depressão em estudantes de medicina da Universidade Federal do Amapá. Revista de Medicina e Saude de Brasilia. 2016; 5(3): 186-99.

28. OSSE CMC, COSTA II. Saude mental e qualidade de vida na moradia estudantil da Universidade de Brasilia. Estudos de Psicologia, 2011; 28(1): 115-122.

29. PARO CA, BITTENCOURT ZZLC. Qualidade de Vida de Graduandos da Área da Saúde. Revista Brasileira de Educação Médica, 2013; 37(3): 365-375.

30. PEREIRA AAG, CARDOSO FMS. Investigando Preditores Psicológicos de Ideação Suicida em Estudantes Universitários. Psicologia: Teoria e Pesquisa, 2018; 33: 1-8.

31. POLONIO IB, et al. Tabagismo entre estudantes de medicina e enfermagem da Universidade Anhembi Morumbi: Prevalência e avaliação da dependência nicotínica e escala de depressão e ansiedade. Arquivos Médicos dos Hospitais e da Faculdade de Ciências Médicas da Santa Casa de São Paulo, 2017; 62(1): 12-17.

32. ROCHA-ALMEIDA LG, FARO A. Levantamento e principais achados de estudos nacionais sobre a depressão - uma revisão sistemática de literature. Revista interdisciplinar de pesquisa e inovação, 2016; 2(1): 1-16.

33. SANTOS KFR, et al. APLICABILIDADE DO INVENTÁRIO DE BECK NOS ACADÊMICOS DE ENFERMAGEM EM UMA INSTITUIÇÃO DE ENSINO DE MINAS GERAIS. Enferm. Foco, 2018; 9 (3): 81-88.

34. SILVA JH, et al. TRANSTORNOS EMOCIONAIS EM ACADÊMICOS DA ÁREA DE SAÚDE: QUAL A INCIDÊNCIA?. Revista Inspirar Movimento e Saúde, 2019; 19(1): 1-17.

35. VASCONCELOS TC, et al. Prevalência de sintomas de ansiedade e depressão em estudantes de medicina. Revista Brasileira de Educação Médica, 2015; 39(1): 135-142

36. VADEBONCOEUR C, et al. Uma meta-análise de ganho de peso em estudantes universitários do primeiro ano: é calouro 15 um mito?. BMC Obes., 2015; 2: 22.

37. VIZZOTTO MM, et al. Saudades de casa: indicativos de depressão, ansiedade, qualidade de vida e adaptação de estudantes universitários. Revista Psicologia e Saúde, 2017; 9(1): 59-73.

38. WERNER MEC, et al. Consumo alcoólico entre universitários. Vamos discutir essa ideia? Interdisciplinar: revista eletrônica da univar. 2015; 13(1): 42-48.

39. YÜKSEL A, BAHADIR-YILMAZ E. Relationship between depression, anxiety, cognitive distortions, and psychological well-being among nursing students. Perspect Psychiatr Care., 2019; 1-7.

40. YOUSEF S, et al. Association between depression and factors affecting career choice among Jordanian nursing students. Frontiers in public health., 2017; 5: 311. 